\title{
The kinetics of water-assisted tautomeric 1,2-proton transfer in azoles: a computational approach
}

\author{
Wojciech P. Oziminski ${ }^{1}$
}

Received: 31 May 2016/ Accepted: 23 July 2016/Published online: 3 August 2016

(c) The Author(s) 2016. This article is published with open access at Springerlink.com

\begin{abstract}
A complete set of azoles undergoing 1,2-proton transfer, consisting of pyrazole, 1,2,3-triazole, 1,2,4-triazole, tetrazole and pentazole, was computationally investigated regarding proton transfer mechanism in gas phase and water solution. Complexes of one azole molecule with 1-4 water molecules were employed to facilitate the proton transfer by lowering the activation energy, which for the isolated azole molecule is prohibitively large. The calculations were performed at the MP2/aug-cc-pVDZ and B3LYP/6-311++G(d,p) levels of theory which showed very good concordance. It follows that in the most probable transition state one azole molecule is accompanied by two water molecules. The activation barrier in water solution modelled by PCM method was lowered to $18.8 \mathrm{kcal} / \mathrm{mol}$ in the case of pyrazole and more for azoles containing more nitrogen atoms in the ring, reaching $6.8 \mathrm{kcal} / \mathrm{mol}$ in the case of pentazole. The analysis of the IRC reaction paths showed that proton transfer in the gas phase has more synchronous character and in the water solution is rather stepwise. It also follows from the analysis of bond lengths in the transition state that in the case of pyrazole the transition state is more cation-like and for other azoles, especially pentazole, is more anion-like. In the water environment, the initial step of proton transfer is moving the proton from azole to the water cluster in all cases
\end{abstract}

Electronic supplementary material The online version of this article (doi:10.1007/s11224-016-0813-y) contains supplementary material, which is available to authorized users.

\section{Wojciech P. Oziminski}

wojozim@gmail.com

1 National Medicines Institute, 30/34 Chełmska Street, 00-725 Warsaw, Poland except pyrazole, where the proton moves first from the water cluster to the azole molecule.

Keywords Proton transfer - Tautomerism - Azoles . Heterocyclic · Theoretical · MP2 - B3LYP - Quantumchemical calculations - Stepwise $\cdot$ Synchronous

\section{Introduction}

Azoles form an important class of aromatic heterocycles [1]. Due to their biological activity, they have found use as building blocks of various drugs: anti-inflammatory (pyrazole) [2], antifungal [3] and sedative [4] (triazole) or antihypertensive [5] (tetrazole) to name only a few examples. Because their rigid rings contain nitrogen atoms with electron lone pairs, they are also attractive as ligands in coordination chemistry $[6,7]$. The presence of both pyrrole-like (acidic) and pyridine-like (basic) atoms in azole rings results in prototropic tautomerism [8], raising the question which tautomer dominates the tautomeric equilibria and by how much. This is important as different tautomers can possess different chemical properties and biological activity [9]. The thermodynamics of azoles tautomerism were studied by many authors with respect to pyrazoles [10, 11], imidazoles [12], triazoles [13-15] and tetrazoles $[16,17]$. However, the kinetic aspects of proton transfer, including the height of the activation barrier, are also important, because the pace, with which the tautomeric equilibria establish, depends on it [18]. Theoretical studies show that the barrier of unassisted proton transfer is very high —in the case of pyrazole, it is $51 \mathrm{kcal} / \mathrm{mol}$ [19] and in the case of tetrazole $49.5 \mathrm{kcal} / \mathrm{mol}$ [20]. However, if proton transfer between azole tautomers is to take place at a reasonable speed at the room temperature, the barrier 
should be $<20 \mathrm{kcal} / \mathrm{mol}$ [8] and this condition can be facilitated by solvent molecules capable of creating hydrogen bonds with azole and acting as helpers. Indeed, the experimental studies in solution show that the actual activation barriers are in range of 10-14 kcal/mol [21-25].

As was pointed out by Alkorta and Elguero [26], the kinetic aspect of azoles' tautomerism was "somewhat neglected" in the past when the focus was on the thermodynamics. This changed recently and there appear an increasing number of publications dealing with theoretical determination of the activation barrier in the case of solvent-assisted proton transfer in various azoles. In one of the first theoretical studies, the barrier for proton transfer in pyrazole assisted by two water molecules was estimated to be $0.48 \mathrm{kcal} / \mathrm{mol}$ at the INDO semiempirical level of theory [27]. In another study, the barrier for proton transfer in tetrazole mediated by an ammonia molecule was calculated as $15.9 \mathrm{kcal} / \mathrm{mol}$ [28]. In a series of papers, Chermahini et al. studied water- and ammonia-assisted proton transfer in thio- and seleno derivatives of various azoles. It was found that the barrier for 1,2-proton transfer in 1H-pyrazole-5-thiol was reduced from 50.1 to $29.5 \mathrm{kcal} / \mathrm{mol}$ at the B3LYP/6-311++G(d,p) level of theory by the assistance of 1 water molecule [29]. In another paper, the tetrazole 5-thione molecule was under consideration and the activation barrier for 1,2-proton transfer was reduced from 53.0 to $23.5 \mathrm{kcal} / \mathrm{mol}$ by 1 water molecule at the B3LYP/6$311++\mathrm{G}(\mathrm{d}, \mathrm{p})$ level of theory [30]. For the tetrazole selenone, the results were similar and 1 water molecule reduced the barrier from 52.8 to $27.3 \mathrm{kcal} / \mathrm{mol}$ at the mPW2PLYP/6-311++G(d,p) level of theory [31]. The authors considered also the ammonia molecule as a helper, and it follows that it lowered the barrier even more, to $17.4 \mathrm{kcal} / \mathrm{mol}$. Further lowering of the activation energy was possible by assistance of two molecules during the proton transfer. In the case of two water molecules, the activation barrier was lowered to $15.5 \mathrm{kcal} / \mathrm{mol}$ and in the case of 1 water and 1 ammonia molecule to $12.0 \mathrm{kcal} / \mathrm{mol}$. Thus, it is apparent that inclusion of a second helper molecule has a strong impact on reducing the barrier which in this case seems to be in the range of previously mentioned experimental results in solution. Other authors had gone even a step further and included a third water molecule as the helper for the proton transfer in keto/amino derivative of 1,3,4-oxadiazole [32]. In the case of proton transfer from the external amino group to the ring nitrogen atom, the first water molecule decreased the barrier from 59.8 to $22.4 \mathrm{kcal} / \mathrm{mol}$ at the B3LYP/6-311++G(d,p) level of theory. The second water molecule lowered the barrier further to $16.7 \mathrm{kcal} / \mathrm{mol}$. The third water molecule had almost no effect. On the other hand, in the case of proton transfer between ring nitrogen atom and the keto group the barrier was raised by the third water molecule. In a very recent paper, the kinetics of proton transfer in a series of 3(5)-substituted pyrazoles was studied using MP2/6$311++\mathrm{G}(\mathrm{d}, \mathrm{p})$ level of theory [33].The barrier for unassisted proton transfer for unsubstituted pyrazole was determined to be $48.3 \mathrm{kcal} / \mathrm{mol}$. It was found that the barrier was lowered to $29.2 \mathrm{kcal} / \mathrm{mol}$ by the assistance of 1 water molecule and to $22.5 \mathrm{kcal} / \mathrm{mol}$ by the assistance of 1 ammonia molecule.

All the above-mentioned studies describe consistently lowering of the activation barrier for proton transfer in particular azoles by subsequent helper molecules. But there is no investigation how the barrier is lowered in a continuous set of azoles from pyrazole to pentazole, e.g. how the aza-substitution of the azole ring influences the barrier. Another question worth answering is: which number of water molecules is actually taking part in the proton transfer in azoles? The preliminary answer from the literature studies can be 2 . But the case of 3 water molecules assisting the proton transfer is definitely not fully investigated, there is only 1 paper where only the proton transfer between external group and the ring was studied, but not the 1,2-proton shift in the ring [31]. Therefore, the main aim of the current project is to theoretically model the transition states for 1,2-proton transfer assisted by 1-4 water molecules for fully representative series of azoles: pyrazole, 1,2,4-triazole, 1,2,3-triazole, tetrazole and pentazole in gas phase and in water solution. This will allow establishing some trends for the kinetics of water-assisted proton transfer in azoles with respect to number of the nitrogen atoms in the azole ring and to number of water molecules assisting proton transfer.

\section{Calculation details}

For all 5 azoles studied, the reactant, product and transition state for proton transfer mediated by 1-4 water molecules were optimized at the ab initio Moller-Plesset MP2/augcc-pVDZ and density Functional B3LYP/6-311++G(d,p) levels of theory. In each case, the frequency check was performed to ensure the proper number of negative eigenvalues: 0 in the case of an energy minimum and 1 in the case of the transition state. The height of the activation barrier was measured as the energy difference between the TS and the most stable tautomer. The zero point vibrational energy (ZPE)-corrected values and Gibbs free energies are additionally presented in Supplementary Information. Transition states were subsequently verified by performing the IRC calculations at the B3LYP level. To simulate the behaviour in water solution, full PCM [34] optimizations were also performed for all studied systems. All calculations were performed by using the Gaussian 09 software [35]. 


\section{Results and discussion}

The six possible paths of 1,2-proton transfer are depicted in Scheme 1. Although the pentazole molecule has not been observed experimentally to date, it was included in the set to fully investigate the influence of aza-substitution on the kinetics of proton transfer in the whole series of azoles.

For pyrazole, 1,2,4-triazole and pentazole, the 1,2-proton transfer results in an identical tautomer. In the case of 1,2,3-triazole, the 1,2-proton transfer leads to a different tautomer. The case of tetrazole is more complicated: two possible proton transfer events are possible-one leading to the same $2 \mathrm{H}$ tautomer (path $4 \mathrm{~b}$, Scheme 1) and second leading to the $1 \mathrm{H}$ tautomer (path $4 \mathrm{a}$, Scheme 1), and thus for this molecule, two distinct transition states were modelled. The activation energy for 1,2,3-triazole and tetrazole was calculated as the energy difference between the transition state and the most stable tautomeric form $2 \mathrm{H}$ at the MP2/aug-cc-pVDZ level (without vibrational/thermal corrections) for clusters with 1-4 water molecules.

The complexes created by a pyrazole molecule and 1-4 water molecules are shown in Fig. 1.

For the other azoles, these structures are similar and they mainly differ in the $\mathrm{N} \cdots \mathrm{HO}$ and $\mathrm{O} \cdots \mathrm{HN}$ hydrogen bond lengths which are presented in Tables 1,2,3 and 4. However, for 1 water molecule there are cases where there is only one hydrogen bond formed between the azole and the water molecule in the reactant complex. In the gas phase, this situation applies only to the pentazole (Tables 1, 2, position designed as N/A), but in the PCM modelled water solution this is the case of all modelled azoles (Tables 3,4). There are two possible arrangements of water molecule and azole connected by 1 hydrogen bond: (a) the bond is formed between azole hydrogen and water molecule oxygen, and (b) the bond is formed between azole nitrogen and water hydrogen atom. The calculations predict that in all cases the structure of type (a) is more stable. The structures and energies of single hydrogen bond complexes of azoles with 1 water molecules are presented in Supplementary Information (Figures 1-2SI).

To answer the question why the pentazole molecule forms only 1 hydrogen bond with 1 water molecule in the gas phase, let us look at the trends in the hydrogen bond length (and thus strength) with the consecutive aza-substitution (Table 1).

It follows from Table 1 that when the number of nitrogen atoms in the ring increases, the $\mathrm{N} \cdots \mathrm{HO}$ bond length is increased, and thus, the bond is weakened. The reverse trend can be observed for the O...HN hydrogen bondwhen the aza-substitution increases, this bond becomes shorter and thus more strong (Table 2). Thus, when moving from pyrazole to pentazole, the $\mathrm{O} \cdots \mathrm{HN}$ hydrogen bond becomes stronger and the $\mathrm{N} \cdots \mathrm{HO}$ bond weaker, and finally, in the case of pentazole the latter ceases to exist.

In the case of water environment, all azoles form only 1 hydrogen bond with 1 water molecule (Tables 3-4). It follows from Table 4 that the $\mathrm{O} \cdots \mathrm{HN}$ bond becomes stronger with aza-substitution. We lack the similar data for $\mathrm{N} \cdots \mathrm{HO}$ bond in Table 3, but if we compare the results for 2-4 water molecule, a trend is revealed: the more nitrogen atoms, the longer and weaker the $\mathrm{N} \cdots \mathrm{HO}$ bond. This explanation is also supported by the comparison of energies of two possible structures (N...HO complex and $\mathrm{O} \cdots \mathrm{HN}$ complex) for various azoles (Figure $2 \mathrm{SI}$ ). For the pyrazole molecule, the $\mathrm{O} \cdots \mathrm{HN}$ bonded structure is only slightly more favourable than $\mathrm{N} \cdots \mathrm{HO}$ bonded one (the energy difference is $0.37 \mathrm{kcal} / \mathrm{mol}$ ). This difference, however, increases as the ring contains more nitrogen atoms and for 1,2,4-triazole is $1.2 \mathrm{kcal} / \mathrm{mol}$, for tetrazole is $3.61 \mathrm{kcal} / \mathrm{mol}$ and for pentazole is $6.32 \mathrm{kcal} / \mathrm{mol}$.

The question arises-why in the gas phase only pentazole molecule formed single hydrogen bond and in the

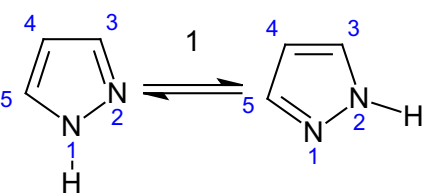

$1 H$-pyrazole

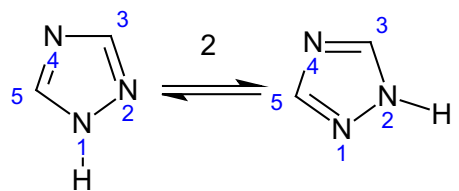

$1 H-1,2,4$-triazole

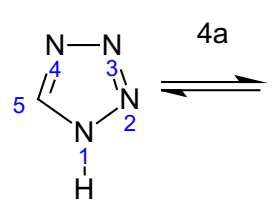

$1 H$-tetrazole

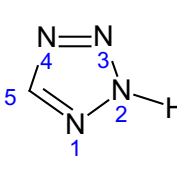

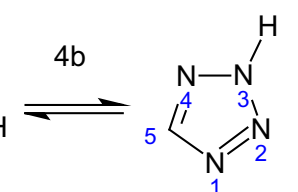

-tetrazole

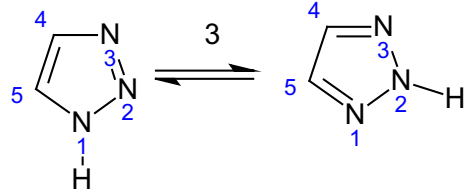

$1 H$-1,2,3-triazole

2H-1,2,3-triazole

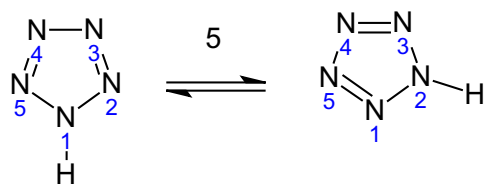

$1 H$-pentazole

Scheme 1 Possible 1,2-proton transfer processes in azoles 


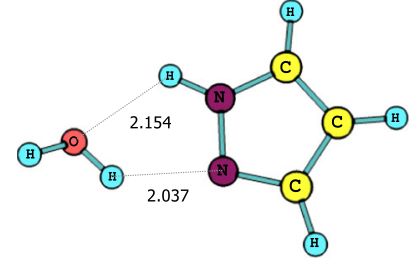

1

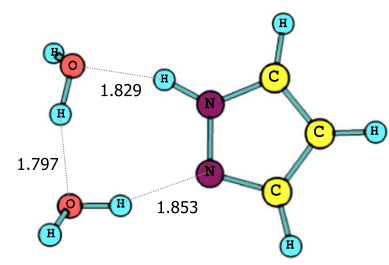

2

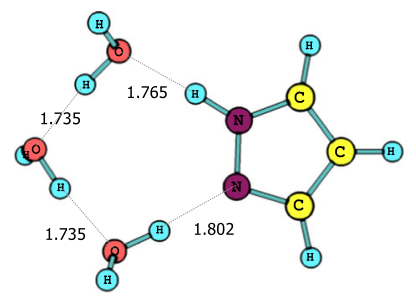

3

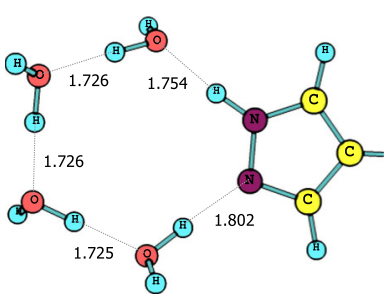

4

Fig. 1 Gas phase MP2 optimized complexes of pyrazole with 1-4 water molecules

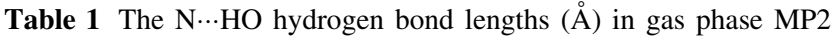
optimized complexes of azoles with 1-4 water molecules

\begin{tabular}{lllll}
\hline Azole & 1 & 2 & 3 & 4 \\
\hline Pyrazole & 2.037 & 1.853 & 1.802 & 1.802 \\
1,2,4-Triazole & 2.154 & 1.899 & 1.841 & 1.841 \\
$2 H-1,2,3$-Triazole & 2.162 & 1.913 & 1.852 & 1.836 \\
$2 H$-Tetrazole $(1 H 2 H)$ & 2.523 & 1.976 & 1.899 & 1.881 \\
$2 H$-Tetrazole $(2 \mathrm{H} 3 \mathrm{H})$ & 2.379 & 1.966 & 1.895 & 1.888 \\
Pentazole & N/A & 2.054 & 1.955 & 1.945
\end{tabular}

Table 2 The $\mathrm{O} \cdots \mathrm{HN}$ hydrogen bond lengths $(\AA)$ in gas phase MP2 optimized complexes of azoles with 1-4 water molecules

\begin{tabular}{lllll}
\hline Azole & 1 & 2 & 3 & 4 \\
\hline Pyrazole & 2.154 & 1.829 & 1.765 & 1.754 \\
1,2,4-Triazole & 2.081 & 1.792 & 1.728 & 1.716 \\
$2 H$ - $1,2,3$-Triazole & 2.108 & 1.789 & 1.713 & 1.700 \\
$2 H$-Tetrazole $(1 H 2 H)$ & 1.965 & 1.735 & 1.662 & 1.648 \\
$2 H$-Tetrazole $(2 H 3 H)$ & 1.998 & 1.735 & 1.661 & 1.642 \\
Pentazole & $1.761^{\mathrm{a}}$ & 1.670 & 1.599 & 1.580 \\
\hline
\end{tabular}

${ }^{a}$ The N...HO hydrogen bond is absent in this structure

Table 3 The N $\cdots$ HO hydrogen bond lengths $(\AA)$ in water solution (PCM) MP2 optimized complexes of azoles with 1-4 water molecules

\begin{tabular}{lllll}
\hline Azole & 1 & 2 & 3 & 4 \\
\hline Pyrazole & N/A & 1.852 & 1.805 & 1.803 \\
1,2,4-Triazole & N/A & 1.915 & 1.853 & 1.851 \\
$2 H-1,2,3$-Triazole & N/A & 1.928 & 1.862 & 1.857 \\
$2 H$-Tetrazole $(1 H 2 H)$ & N/A & 2.024 & 1.920 & 1.907 \\
$2 H$-Tetrazole $(2 H 3 H)$ & N/A & 2.021 & 1.915 & 1.906 \\
Pentazole & N/A & 2.180 & 1.991 & 1.978 \\
\hline
\end{tabular}

water environment all four representative azoles have done so? We can answer this question by comparing the results for 2 water molecules. It follows from comparison of
Table 4 The $\mathrm{O} \cdots \mathrm{HN}$ hydrogen bond lengths $(\AA)$ in water solution (PCM) MP2 optimized complexes of azoles with 1-4 water molecules

\begin{tabular}{lllll}
\hline Azole & 1 & 2 & 3 & 4 \\
\hline Pyrazole & $1.837^{\mathrm{a}}$ & 1.849 & 1.771 & 1.750 \\
$1,2,4$-Triazole & $1.789^{\mathrm{a}}$ & 1.790 & 1.719 & 1.700 \\
$2 H-1,2,3$-Triazole & $1.777^{\mathrm{a}}$ & 1.779 & 1.707 & 1.689 \\
$2 H$-Tetrazole $(1 H 2 H)$ & $1.714^{\mathrm{a}}$ & 1.699 & 1.632 & 1.624 \\
$2 H$-Tetrazole $(2 H 3 H)$ & $1.714^{\mathrm{a}}$ & 1.699 & 1.633 & 1.618 \\
Pentazole & $1.633^{\mathrm{a}}$ & 1.599 & 1.536 & 1.520 \\
\hline
\end{tabular}

${ }^{a}$ The $\mathrm{N} \cdots \mathrm{HO}$ hydrogen bond is absent in this structure

Tables 1 and 3 that in the water solution the $\mathrm{N} \cdots \mathrm{HO}$ bond is longer and thus weaker. This effect becomes more pronounced with more nitrogen atoms in the ring. Thus, we can assume that in the gas phase only in the case of pentazole this bond is too weak to exist, but in the water environment it is too weak for all azoles. On the contrary, the $\mathrm{O} \cdots \mathrm{HN}$ bond (Tables 2,4) is considerable shorter (and stronger) in water environment. This is most clearly seen in the case of 1 water molecule, and this difference becomes smaller for more water molecules.

1. The dependence of the activation barrier height and the nature of the transition state on the number of assisting water molecules

The results of MP2/aug-cc-pVDZ calculations are shown in Tables 5 and 6 for gas phase and water solution accordingly. It follows that the activation barrier is always highest in the case of 1 water molecule. This can be rationalized taking pyrazole as an example (Fig. 2).

In the transition state, between azole and water molecules a pseudo-ring is formed including hydrogen bonds (Fig. 2). It follows that these hydrogen bonds are farthest from the linearity in case of 1 water molecule, which destabilizes the TS. Bond angles in case of 2 water molecules are much closer to linearity which is reflected in lowering the activation barrier by about $10-13 \mathrm{kcal} / \mathrm{mol}$. This effect increases as the number of nitrogen atoms in the ring is increased. The second reason for barrier lowering 
Table 5 MP2 gas phase activation energy for proton transfer in azoles according to number of water molecules involved

\begin{tabular}{lllll}
\hline Azole & $1 \mathrm{w}$ & $2 \mathrm{w}$ & $3 \mathrm{w}$ & $4 \mathrm{w}$ \\
\hline Pyrazole & 27.61 & 19.51 & 20.97 & 25.35 \\
124-Tria & 27.58 & 19.62 & 20.82 & 24.38 \\
123-Tria & 29.58 & 20.71 & 21.34 & 24.22 \\
Tetra1H2H & 28.17 & 18.47 & 18.05 & 19.80 \\
Tetra2H3H & 27.11 & 17.48 & 17.14 & 19.37 \\
Penta & 26.16 & 14.32 & 12.84 & 14.09 \\
\hline
\end{tabular}

Energy in $\mathrm{kcal} / \mathrm{mol}$ calculated with respect to $2 H$ tautomer

Table 6 MP2 water solution (PCM) activation energy for proton transfer in azoles according to number of water molecules involved

\begin{tabular}{lllll}
\hline Azole & $1 \mathrm{w}$ & $2 \mathrm{w}$ & $3 \mathrm{w}$ & $4 \mathrm{w}$ \\
\hline Pyrazole & 29.49 & 18.78 & 18.78 & 20.51 \\
124-Tria & 27.62 & 17.99 & 17.10 & 18.31 \\
123-Tria & 28.09 & 18.02 & 16.87 & 17.87 \\
Tetra1H2H & 24.27 & 12.86 & 10.72 & 11.03 \\
Tetra2H3H & 24.53 & 13.00 & 10.81 & 11.11 \\
Penta & 19.53 & 6.85 & 4.04 & 3.50 \\
\hline
\end{tabular}

Energy in $\mathrm{kcal} / \mathrm{mol}$ calculated with respect to $2 H$ tautomer

can be better delocalization of electronic charge in the transition state with increasing number of water molecules. In the transition state, the azole molecule possesses some charge depending on the number of nitrogen atoms in the ring. For pyrazole and 1,2,4-triazole, the azole in the transition state is more cation-like and for 1,2,3-triazole, tetrazole and pentazole more anion-like. This is because of high electronegativity and thus electron-withdrawing properties of the nitrogen atoms. Regardless of the sign of the charge, the more water molecules assist in the proton transfer, the better delocalization of this charge in the transition state and thus the lower the activation barrier.

The effect of introduction of third and fourth water molecule depends on the kind of azole and environment. First let us analyse the gas phase results (Table 5).
In the case of pyrazole, 1,2,4- and 1,2,3-triazole, the third water molecule slightly increases the activation energy. In case of the tetrazole, the third water molecule lowers the barrier by $0.42 \mathrm{kcal} / \mathrm{mol}$ and in case of pentazole by $1.5 \mathrm{kcal} / \mathrm{mol}$. Introduction of fourth water molecule raises the activation barrier for all studied systems, and this effect is largest for pyrazole and decreases when moving to pentazole.

Now, let us compare the water solution results (Table 6). Here, the introduction of third water molecule does not change the barrier for pyrazole and 1,2,4-triazole, but for 1,2,3-triazole the barrier is lowered by $1.15 \mathrm{kcal} /$ mol. The effect is consistent down the series and for tetrazole, especially pentazole, the lowering of the barrier is large. Introduction of fourth water molecule causes the increase in the activation barrier for all azoles except pentazole. Very low activation barriers for pentazole in water solution can be attributed to highly anion-like character of the transition state of the pentazole, which is strongly stabilized in highly polar solvent-water (simulated here by the PCM methodology).

Summarizing, the lowest activation barriers are observed for transfers mediated by two and three water molecules. Four water molecules would be preferred only in the case of pentazole in water solution, but for statistical reasons this number of molecules arranged properly in the transition state is highly improbable. Thus, the answer to the question which number of water molecules is "optimal" depends on particular azole. As the number of nitrogen atoms in the ring increases, the preference shifts from 2 water molecules to 3 . However, for statistical reasons the transition states involving less molecules are more probable so the conclusion is that the most probable transition state involves 2 water molecules.

2. The dependence of the activation barrier height and the nature of the transition state on the number of nitrogen atoms in the ring in the case of 2 water molecules

It follows that aza-substitution causes barrier lowering which can be clearly seen in tetrazole and pentazole results, especially in the case of water solution (Tables 5, 6). The structure of the transition state depends on the number of

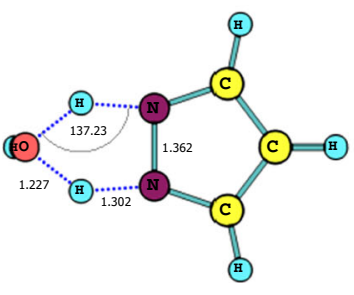

1

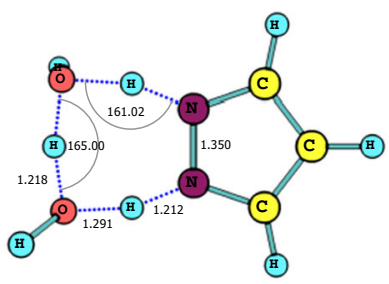

2

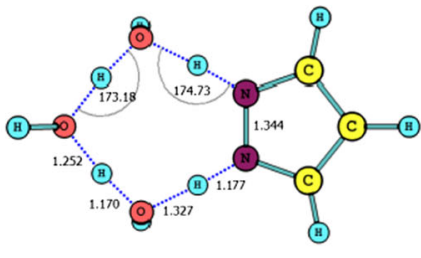

3

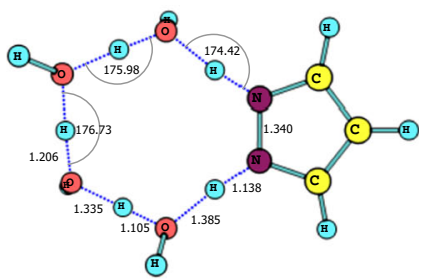

4

Fig. 2 Gas phase MP2 optimized transition states for 1,2-proton transfer in complexes of pyrazole with 1-4 water molecules 
nitrogen atoms in the ring. In the case of pyrazole, it is cation-like which means that both prototropic hydrogen atoms are close to the azole molecule. When the number of nitrogen atoms increases, the transition state becomes more anion-like which means that both prototropic hydrogen atoms are more distant to the azole molecule and closer to the water molecules cluster. This situation is shown in Fig. 3. We have chosen only transition states of pyrazole, 1,2,4-triazole, tetrazole $(2 \mathrm{H} 3 \mathrm{H}$ transition state) and pentazole for showing the evolution in hydrogen bond lengths because these rings are symmetrical and well illustrate the described tendencies.

As mentioned previously, this tendency is caused by the electron-withdrawing character of pyridine-like nitrogen atoms in the ring. The more nitrogen atoms in the ring, the more the electronic charge is withdrawn from the two nitrogen atoms engaged in proton transfer into the rest of the ring, and the bonding between the nitrogen atoms and the proton become weaker and the transition state of azole becomes more anion-like. Conversely, the distance between hydrogen and water oxygen becomes smaller. This apparently facilitates proton transfer, and the activation energy especially for pentazole is much smaller than for pyrazole. This tendency is even stronger in the case of water environment.

3. The dependence of the nature of the transition state on the environment

Gas phase vs water solution. Analysis of the IRC paths (provided in a following paragraph) and of the imaginary vibrational modes of the transition states reveals that in the gas phase the proton transfer is more synchronous, but in the water solution modelled by PCM method the proton transfer is more stepwise and the imaginary vibration corresponds mostly to the proton shift between two water molecules. The proton transfer in complex of pyrazole with 2 water molecules in gas phase and water solution animated via IRC path is presented in the form of two animated GIF pictures as Supplementary Data. From these animations, the synchronous vs stepwise modes can be clearly seen. The second trend observed is that pure synchronous transfer occurs only in the case of pyrazole in the gas phase. When the number of hydrogen atoms in the ring increases, the transition state becomes more anion-like as described previously and the proton transfer becomes partially stepwise. However, in the case of water environment for all azoles the stepwise mechanism is observed. The difference in mode of the proton transfer can be described in the following way. For pyrazole, the transition state in cation-like, so at the beginning of the transfer a proton moves from the water cluster to azole, next step is from one water molecule to the other, and the last step is from azole cation to water molecule. But in the case of all other azoles the proton travels first from the azole to the water cluster, forming an anion-like transition state, and then travels between water molecules and finally from water to the azole.

\section{IRC reaction paths}

As mentioned in "Calculational details" section, the IRC paths for all proton transfer events in gas phase and water solution were modelled at the B3LYP level to avoid time-consuming MP2 calculations. In all cases, the calculations proved that the transition state indeed connects the proper reactants and products. The calculations of IRC paths shed some light on the nature of proton transfer which was especially helpful for comparison of gas phase vs water solution transfers. In Figs. 4 and 5, only the border cases, pyrazole and pentazole, are shown to show the impact of aza-substitution. The most statistically probable clusters with 2 water molecules were chosen. From the first look, it is obvious that these figures present very different pictures. Several conclusions are drawn in Figs. 4 and 5.

Firstly, in the gas phase both azoles show near cubic curvatures near the transition state; however, in the case of pentazole, this region is much more flat. As was previously shown, in this case the transition state is more anion-like and the transferable hydrogen atoms are more distant to the

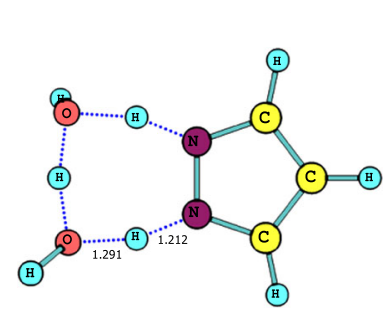

Pyrazole TS

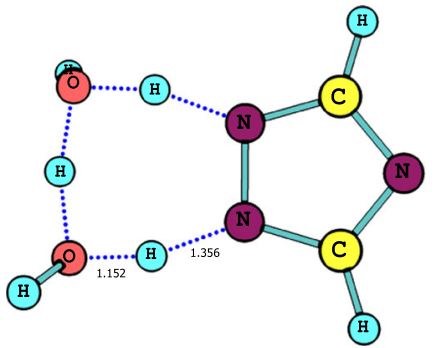

1,2,4-triazole TS

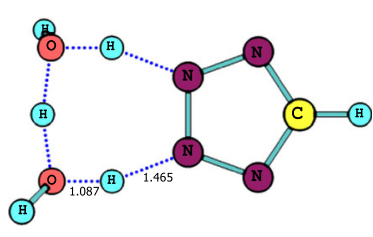

Tetrazole $2 \mathrm{H} 3 \mathrm{H}$ TS

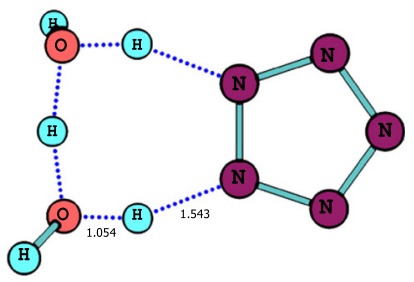

Pentazole TS

Fig. 3 Hydrogen-nitrogen and hydrogen-oxygen distances in transition state structures with two water molecules optimized in the gas phase at the MP2/aug-cc-pVDZ level 


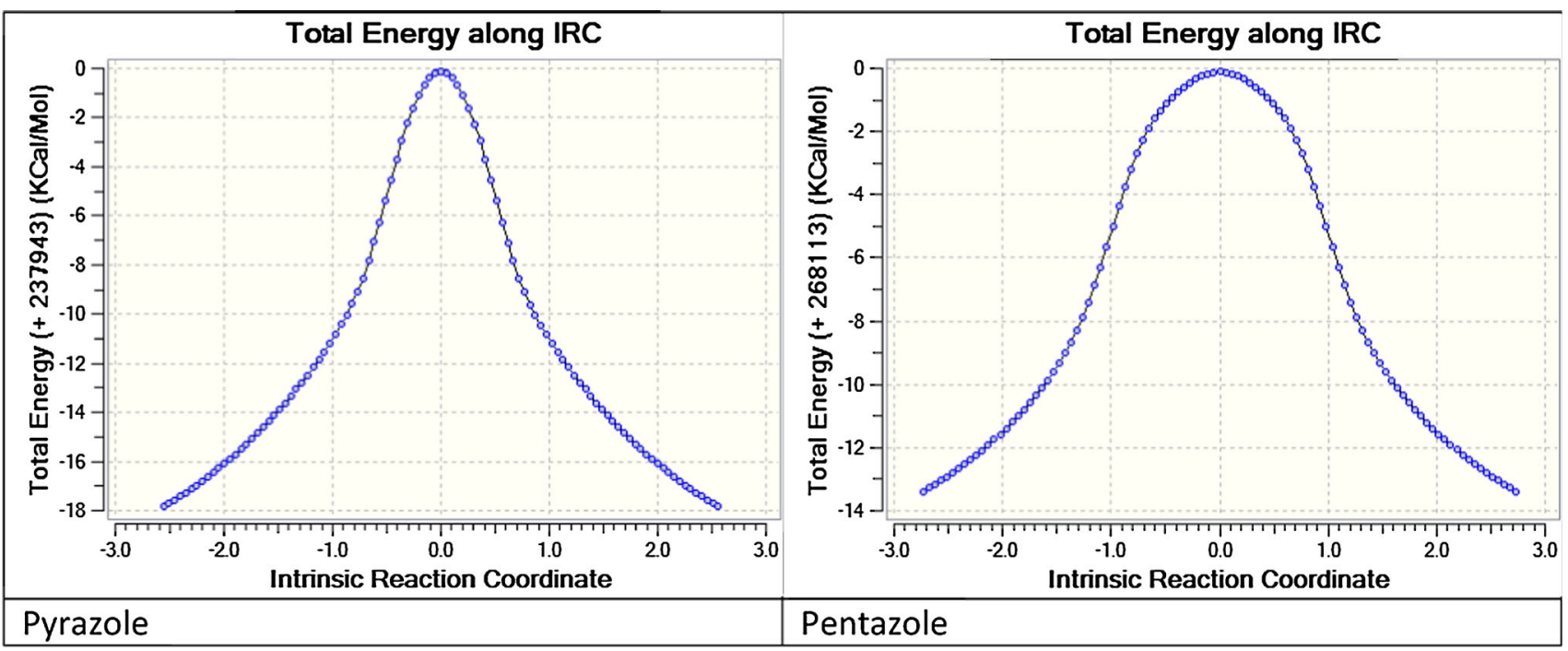

Fig. 4 IRC paths of proton transfer in pyrazole and pentazole clusters with 2 water molecules in gas phase

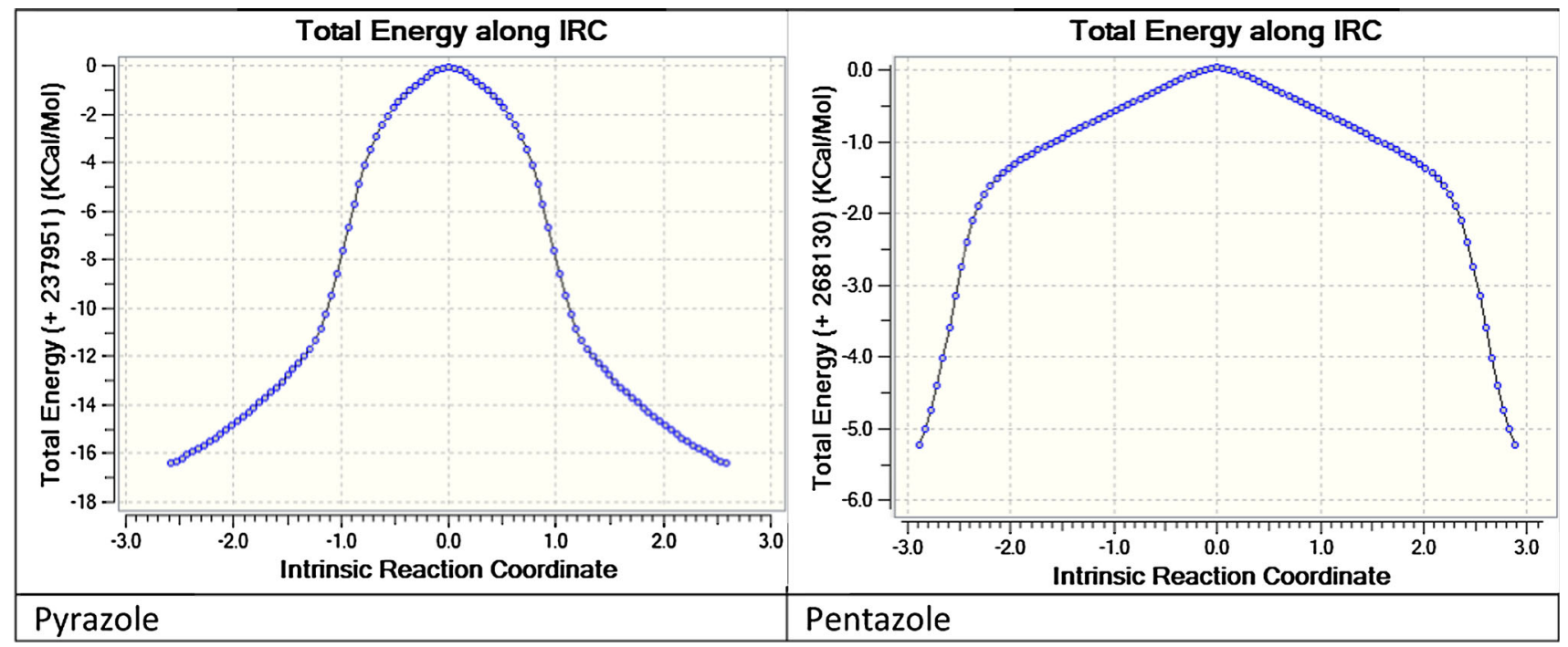

Fig. 5 IRC paths of proton transfer in pyrazole and pentazole clusters with 2 water molecules in water solution

azole molecule which may account for this effect. The proton transfer in the gas phase has synchronous character, and both protons move simultaneously. Secondly, in the water solution, the curvature near transition state is rather linear, which is especially pronounced in the case of pentazole. This corresponds to more stepwise mechanism in the case of water solution where one proton is first transferred to the azole forming a cation, and then, the second proton moves from one water molecule to the other, and at the end, the third proton moves from the second protonation site of azole to the cluster of water molecules. Also in the case of water environment, a flattening of the peak when comparing pyrazole with pentazole can be observed. This effect is smaller but shows that the more nitrogen atoms in the ring, the less steep the IRC path curve is, which corresponds to lower activation barrier.

5. Imaginary frequencies of the transition states

The imaginary frequencies of the transition states are gathered in Tables 7 and 8.

Regarding the number of water molecules, it follows that the more water molecules are involved, the less negative is the imaginary frequency in the gas phase, and in the water solution, this is true up to 3 water molecules. This tendency can be explained by simply noticing that when the number of water molecules increases, the transition state becomes larger and more floppy and it is natural that the absolute value of the frequency is lowered. Regarding 
the number of nitrogen atoms, the tendency is similar-the more nitrogen atoms in the ring, the less negative the frequency. This time it can be rationalized by keeping in mind that the transition state becomes more anion-like, so the actual proton transfer takes place in the cluster of water molecules especially in the water environment where it has a stepwise character. And finally, regarding the

Table 7 Imaginary frequency corresponding to the proton transfer in the transition state calculated at the MP2/aug-cc-pVDZ level in the gas phase

\begin{tabular}{lllll}
\hline Azole & $1 \mathrm{w}$ & $2 \mathrm{w}$ & $3 \mathrm{w}$ & $4 \mathrm{w}$ \\
\hline Pyrazole & -1746.45 & -1538.41 & -1377.46 & -1092.43 \\
124-Tria & -1482.06 & -1467.75 & -1199.3 & -816.03 \\
123-Tria & -1425.16 & -1405.51 & -1111.31 & -727.34 \\
Tetra & -980.12 & -1022.82 & -597.33 & -533.44 \\
Tetra2H3H & -926.83 & -1022.32 & -626.55 & -579.35 \\
Penta & -580.31 & -754.07 & -316.39 & -448.91 \\
\hline
\end{tabular}

Table 8 Imaginary frequency corresponding to the proton transfer in the transition state calculated at the MP2/aug-cc-pVDZ level in water solution

\begin{tabular}{lllll}
\hline Azole & $1 \mathrm{w}$ & $2 \mathrm{w}$ & $3 \mathrm{w}$ & $4 \mathrm{w}$ \\
\hline Pyrazole & -1220.08 & -1171.64 & -152.08 & -886.06 \\
124-Tria & -505.65 & -973.32 & -141.16 & -580.53 \\
123-Tria & -459.75 & -947.24 & -140.95 & -575.28 \\
Tetra & -329.15 & -767.56 & -298.01 & -541.78 \\
Tetra2H3H & -311.69 & -763.92 & -296.35 & -549.24 \\
Penta & -270.23 & -644.12 & -556.78 & -518.57 \\
\hline
\end{tabular}

environment it can be seen that in most cases the imaginary frequency is lowered in the water environment comparing to the gas phase, which can be explained by general lengthening of the bond lengths by the water environment simulated by the PCM method.

6. The dipole moment in the studied systems

A parameter which could help to understand the dependencies between the activation energy and the number of water molecules or number of nitrogen atoms is the dipole moment of the azole-water cluster. Thus, the MP2 dipole moments were calculated for the gas phase and water solution and are presented in Tables 9 and 10 .

Dipole moment for the transition state is almost always larger than for the reactant because of larger separation of tautomeric proton and azole molecule in the TS. There is also a consistent trend that as the aza-substitution increases, the dipole moment becomes larger because the negative charge is more and more concentrated in the azole ring. Also the ring-proton distance becomes larger and larger. The largest dipole moment is observed for the pentazole where the ring contains only the electronegative nitrogen atoms and the ring-proton separation is largest. The general trend is that the higher the dipole moment of the TS is, the lower is the energy of activation. The activation barrier is almost always lower in solution and more polar structure and larger dipole moment of the TS contribute to this phenomenon. This is most clear for tetrazole and pentazole molecules.

7. MP2 and B3LYP methods comparison

The thermodynamic data obtained by MP2 and B3LYP methods are in very good concordance. For the most
Table 9 MP2 gas phase dipole moments of $2 \mathrm{H}$ tautomer and transition state
Table 10 MP2 water solution (PCM) dipole moments of $2 \mathrm{H}$ tautomer and transition state

\begin{tabular}{lllllllll}
\hline Azole & 1w-taut & 1w-TS & 2w-taut & 2w-TS & 3w-taut & 3w-TS & 4w-taut & 4w-TS \\
\hline Pyrazole & 1.58 & 1.54 & 1.02 & 2.28 & 1.37 & 3.45 & 0.79 & 5.43 \\
124-Tria & 2.36 & 3.43 & 1.87 & 2.93 & 2.22 & 4.59 & 1.92 & 6.91 \\
123-Tria & 2.02 & 3.25 & 1.80 & 2.86 & 2.06 & 3.99 & 1.15 & 5.31 \\
Tetra1H2H & 4.30 & 6.24 & 3.68 & 6.33 & 3.82 & 7.80 & 3.45 & 8.64 \\
Tetra2H3H & 2.30 & 4.11 & 1.52 & 4.12 & 1.77 & 5.76 & 1.31 & 7.43 \\
Penta & 7.18 & 7.75 & 4.90 & 8.01 & 4.85 & 9.60 & 4.64 & 11.12 \\
\hline
\end{tabular}

\begin{tabular}{lrrlrlrrr}
\hline Azole & 1w-taut & 1w-TS & 2w-taut & 2w-TS & 3w-taut & 3w-TS & 4w-taut & 4w-TS \\
\hline Pyrazole & 3.66 & 2.62 & 1.66 & 5.94 & 2.12 & 8.88 & 1.52 & 9.59 \\
124-Tria & 5.94 & 7.21 & 2.37 & 6.71 & 2.83 & 9.60 & 2.62 & 11.70 \\
123-Tria & 2.75 & 6.26 & 2.27 & 5.79 & 2.86 & 8.32 & 4.49 & 10.06 \\
Tetra1H2H & 10.50 & 10.49 & 4.50 & 10.09 & 4.80 & 11.82 & 4.01 & 14.04 \\
Tetra2H3H & 5.07 & 7.74 & 1.88 & 7.29 & 2.39 & 9.40 & 1.91 & 11.40 \\
Penta & 8.46 & 12.33 & 6.18 & 11.93 & 6.18 & 13.26 & 5.96 & 15.56 \\
\hline
\end{tabular}


important case-complexes with 2 water molecules, the correlation coefficient equals to 0.995 for gas phase and 0.990 for the water solution. The mean energy difference between activation energies calculated by these two methods is about $0.32 \mathrm{kcal} / \mathrm{mol}$ for gas phase and $0.68 \mathrm{kcal} / \mathrm{mol}$ for water solution. This is a very small value, well below $1 \mathrm{kcal} / \mathrm{mol}$, and thus, the conclusions drawn from both methods should be consistent, and the results derived from the less expensive B3LYP calculations can be trusted for these systems.

\section{Conclusions}

1. The proton transfers in complexes of azole molecule with 1-4 water molecules were investigated computationally

2. In the complexes of azole molecule with one water molecule in water environment, the calculations show that there is only one hydrogen bond between azole and water. In complexes with 2-4 water molecules, there are always two hydrogen bonds.

3. Transition states for proton transfers with two and three "helper" water molecules have similar activation energies but for statistical reasons more probable is the case with two water molecules.

4. The addition of second "helper" water molecules lowers the activation barrier much (about $10 \mathrm{kcal} / \mathrm{mol}$ ) comparing to complexes with 1 water molecule. Addition of additional water molecule causes small effect, and such transition state is less probable for statistical reasons.

5. The pyrazole molecule in the transition state has some cationic character: both prototropic hydrogen atoms are close to the azole ring. Increasing the number of nitrogen atoms in the ring increases the distance between hydrogen atoms and the azole ring, thus making the azole molecule in the transition state more anionic, which is accompanied by lowering of the activation barrier.

6. In the gas phase, the proton transfer is more synchronous, and in the water environment, it is more stepwise.

7. In the process of proton transfer, the proton moves first from azole molecule to the water cluster in all cases except pyrazole, which forms cation-like transition state and the proton moves first from the water molecule to the azole.

8. The presence of water environment lowers the activation barriers.

9. The results of B3LYP/6-311++G(d,p) calculations are consistent with MP2/aug-cc-pVDZ calculations
Acknowledgments Computational Grant G36-9 from the Interdisciplinary Centre for Mathematical and Computational Modelling at Warsaw University (ICM UW) is gratefully acknowledged.

Open Access This article is distributed under the terms of the Creative Commons Attribution 4.0 International License (http://crea tivecommons.org/licenses/by/4.0/), which permits unrestricted use, distribution, and reproduction in any medium, provided you give appropriate credit to the original author(s) and the source, provide a link to the Creative Commons license, and indicate if changes were made.

\section{References}

1. Katritzky AR, Ramsden CA, Joule JA, Zhdankin VV (2010) Handbook of heterocyclic chemistry, 3rd edn. Pergamon, New York

2. Francisco ME, Seltzman HH, Gilliam AF, Mitchell RA, Rider SL, Pertwee RG, Stevenson LA, Thomas BF (2002) J Med Chem 45:2708-2719

3. Lass-Florl C (2011) Drugs 71:2405-2419

4. Lobo BL, Greene WL (1997) Ann Pharmacother 31:625-632

5. Le Bourdonnec B, Meulon E, Yous S, Goossens J-F, Houssin R, Henichart J-P (2000) J Med Chem 43:2685

6. Aromía G, Barriosa LA, Roubeaub O, Gameza P (2011) Coord Chem Rev 255:485-546

7. Bai SQ, Young DJ, Hor TS (2011) Chem Asian J 6:292-304

8. Elguero J, Katritzky AR, Denisko OV (2000) Adv Heterocycl Chem 76:1-84

9. Jain KK (2000) Expert Opin Investig Drugs 9:829-840

10. Jaronczyk M, Dobrowolski JC, Mazurek AP (2004) J Mol Struct (Theochem) 673:17-28

11. Alkorta I, Elguero J, Liebman JF (2006) Struct Chem 17:439-444

12. Kurzepa M, Dobrowolski JC, Mazurek AP (2001) J Mol Struct 565-566:107-113

13. Oziminski WP, Dobrowolski JC, Mazurek AP (2004) J Mol Struct (Theochem) 680:107-115

14. Oziminski WP, Dobrowolski JC, Mazurek AP (2003) J Mol Struct 652-653:697-704

15. Oziminski WP (2013) Tetrahedron 69:3197-3205

16. Mazurek AP, Sadlej-Sosnowska N (2000) Chem Phys Lett 330:212-218

17. Trifonov RE, Alkorta I, Ostrovskii VA, Elguero J (2004) J Mol Struct (THEOCHEM) 668:123-132

18. Raczynska ED, Kosinska W, Osmialowski B, Gawinecki R (2005) Chem Rev 105:3561-3612

19. Alkorta I, Elguero J (1998) J Chem Soc Perkin Trans 2:2497-2504

20. Wong MW, Leung-Toung R, Wentrup C (1993) J Am Chem Soc 115:2465-2472

21. Hirota N, Sumitani M, Yoshihara K (1985) J Phys Chem 89:399-401

22. Roumestant ML, Viallefont P, Elguero J, Jacquier R (1969) Tetrahedron Lett 10:495-498

23. Chenon MT, Coupry C, Grant DM, Pugmire RJ (1977) J Org Chem 42:659-661

24. Claramunt RM, Elguero J, Marzin C, Seita J (1979) Anal Quim 75:701-706

25. Perrin M, Thozet A, Cabildo P, Claramunt RM, Valentí E, Elguero J (1993) Can J Chem 71:1443-1449

26. Alkorta I, Elguero J (2009) Top Heterocycl Chem 19:155-202

27. Catalan J, de Paz JLG, Sanchez-Cabezudo M, Elguero J (1986) Bull Soc Chim Fr 429 
28. Alkorta I, Rosas I, Elguero J (1998) J Chem Soc Perkin Trans 2:2671-2675

29. Beni AS, Chermahini ZJ (2013) Struct Chem 24:1713-1723

30. Chermahini AN, Teimouri A, Beni AS (2011) Struct Chem 22:175-181

31. Chermhini AN, Abedi M, Farrokhpour H, Teimouri A, Reisi B (2013) J Mol Model 19:4377-4386

32. Chahkandi B, Tayyari SF, Bakhshaei M, Chahkandi M (2013) J Mol Graph Model 44:120-128

33. Chermahini AN, Teimouri A (2014) J Chem Sci 126:273-281

34. Scalmani G, Frisch MJ (2010) J Chem Phys 132:114110-114115

35. Frisch MJ, Trucks GW, Schlegel HB, Scuseria GE, Robb MA, Cheeseman JR, Scalmani G, Barone V, Mennucci B, Petersson
GA, Nakatsuji H, Caricato M, Li X, Hratchian HP, Izmaylov AF, Bloino J, Zheng G, Sonnenberg JL, Hada M, Ehara M, Toyota K, Fukuda R, Hasegawa J, Ishida M, Nakajima T, Honda Y, Kitao O, Nakai H, Vreven T, Montgomery JA Jr, Peralta JE, Ogliaro F, Bearpark M, Heyd JJ, Brothers E, Kudin KN, Staroverov VN, Kobayashi R, Normand J, Raghavachari K, Rendell A, Burant JC, Iyengar SS, Tomasi J, Cossi M, Rega N, Millam JM, Klene M, Knox JE, Cross JB, Bakken V, Adamo C, Jaramillo J, Gomperts R, Stratmann RE, Yazyev O, Austin AJ, Cammi R, Pomelli C, Ochterski JW, Martin RL, Morokuma K, Zakrzewski VG, Voth GA, Salvador P, Dannenberg JJ, Dapprich S, Daniels AD, Farkas O, Foresman JB, Ortiz JV, Cioslowski J, Fox DJ (2009) Gaussian 09, Revision B.01. Gaussian, Wallingford, CT 